\title{
Enantiomeric separation of amino acid derivatives by non-aqueous capillary electrophoresis using quinine and related compounds as chiral additives
}

\author{
V. Piette, ${ }^{1 *}$ M. Fillet, ${ }^{2}$ W. Lindner ${ }^{3}$ and J. Crommen ${ }^{2}$ \\ ${ }^{1}$ Laboratory of Drug Analysis, Institute of Public Health — Louis Pasteur, Rue J. Wytsman 14, B-1050 Bruxelles, Belgium \\ ${ }^{2}$ Department of Analytical Pharmaceutical Chemistry, Institute of Pharmacy, University of Liège, CHU, B36, B-4000 Liège 1, Belgium \\ ${ }^{3}$ Institute of Analytical Chemistry, University of Vienna, Währingerstrasse 38, A-1090 Vienna, Austria
}

Received 21 June 1999; accepted 18 July 1999

\section{INTRODUCTION}

Non-aqueous capillary electrophoresis (NACE), employing small amounts of well-characterized chiral additive, offers an attractive alternative for screening potential chiral stationary phase selectors and studying the interactions between the selector and various analytes. In a previous work (Piette et al., 1998), a NACE system using a background electrolyte made from $12.5 \mathrm{mM}$ of ammonium acetate in methanol was found to be useful for the investigation of the potential of quinine and tertbutyl carbamoylated quinine as selectors (SOs) for the enantioseparation of various kinds of $\mathrm{N}$-protected amino acids. The influence of a series of parameters on the enantioseparation of the anionic amino acid derivatives by formation of ion pairs with the cationic chiral selectors in the non-aqueous electrolyte was investigated, using uncoated fused silica capillaries: the composition and concentration of the background electrolyte, the concentration of the chiral selector used as counter-ion, the nature and proportions of organic solvents, the capillary temperature and the applied voltage. High selectivity and resolution were obtained with a background electrolyte made from a mixture of $12.5 \mathrm{mM}$ ammonia and $100 \mathrm{mM}$ octanoic acid, containing the chiral selector at $10 \mathrm{~mm}$ concentration in an ethanol-methanol mixture (60:40; $\mathrm{v} / \mathrm{v})$.

In this study, under these operating conditions, 3,5dinitrobenzoyl (DNB) derivatives of leucine (Leu) and phenylalanine (Phe) were examined with respect to selectivity, resolution and migration order for their enantiomers. Quinine (QN), quinidine (QD), cinchonine $(\mathrm{CN})$, cinchonidine (CD), tert-butyl carbamoylated quinine (tBuCQN), tert-butyl carbamoylated quinidine (tBuCQD), dinitrophenyl carbamoylated quinine

*Correspondence to: V. Piette, Laboratory of Drug Analysis, Institute of Public Health - Louis Pasteur, Rue J. Wytsman 14, B-1050 Bruxelles, Belgium.
(DNPCQN) and cyclohexyl carbamoylated quinine (cHexCQN) (cf. Fig. 1) were tested successively as chiral counter ions.

\section{Chiral selectors}

Quinine (QN)<smiles>C=C[C@H]1CN(C(=O)c2ccnc3ccc(OC)cc23)CCN1C</smiles>

Cinchonine $(\mathrm{CN})$<smiles>C=C[C@H]1CN2CC[C@H]1C[C@H]2C(=O)c1ccnc2ccccc12</smiles>

Quinidine (QD)<smiles>C=C[C@@H]1C[C@@H]2CCN(C(=O)c3ccnc4ccc(OC)cc34)C[C@@H]2C1</smiles>

Cinchonidine (CD)<smiles>C=C[C@H]1CN2CC[C@H]1C[C@H]2C(O)(O)c1ccnc2ccccc12</smiles>

Carbamoylated quinine derivatives (CQN)<smiles>[R]NC(=O)O[C@H](c1ccnc2ccc(OC)cc12)[C@H]1CC[C@H]2CN1CC2C=C</smiles>

R : tert-butyl ( $(\mathrm{BuCQN})$ dinitrophenyl (DNPCQN) cyclohexyl (cHexCQN)

Figure 1. Chiral selectors. 


\section{MATERIAL AND METHODS}

All experiments were performed on a Spectraphoresis 1000 CE instrument (Spectraphysics, San Jose, CA, USA) equipped with an automatic injector, an autosampler, a UV/visible detector (190-800 nm) and a temperature control system $\left(15-60^{\circ} \mathrm{C}\right)$. QN was obtained from Sigma (St Louis, MO, USA); QD, CN and CD were from Buchler (Brannschweig, Germany). TBuCQN, tBuCQD, DNPCQN and cHexCQN were synthesized according to a standard procedure described elsewhere (Lindner et al., 1997). The organic solvents were HPLC grade: ethanol absolute from Merck (Darmstad, Germany) and methanol from Fisher Scientific (Leicestershire, UK). Octanoic acid was from Sigma and ammonia solution 25\% from Carlo Erba (Rodano, Italy). The racemic and enantiomerically pure amino acids leucine (Leu) and phenylalanine (Phe) were purchased from Sigma. The DNB-derivative of Phe was synthesized according to a standard derivatization procedure (Kleidernigg et al., 1996), while DNB-Leu was obtained from Sigma. The sample solutions were prepared by dissolving each amino acid derivative at a concentration of $50 \mu \mathrm{g} /$ $\mathrm{mL}$ in methanol. Benzylic alcohol from Sigma $(0.01 \%$ methanolic solution) was used as neutral marker to visualize the electroosmotic flow $\mu_{\mathrm{EOF}}$. Buffers and samples were filtered through a Polypure polypropylene membrane filter $(0.2 \mu \mathrm{m})$ from Alltech (Laarne, Belgium) before use.

Electrophoretic separations were carried out with uncoated fused-silica capillaries, $50 \mu \mathrm{m}$ i.d. and $44 \mathrm{~cm}$ length $(37 \mathrm{~cm}$ to the detector), provided by Supelco (Bellefonte, PA, USA). The injections were made at the cathodic side and the applied voltage was $-25 \mathrm{kV}$ (reversed polarity mode). The normal polarity mode $(+25 \mathrm{kV})$ was used to measure the cathodic electroosmotic flow $\mu_{\mathrm{EOF}}(i \approx 6.5 \mu \mathrm{A})$. The UV detection (at the anodic side) was performed at $214 \mathrm{~nm}$. Injections were made in the hydrodynamic mode for a period of $5 \mathrm{~s}$ (corresponding to $13.3 \mathrm{~nL}$ ) and the capillary was thermostatted at $15^{\circ} \mathrm{C}$. The resolution $(R s)$ was calculated according to the standard expressions based on peak width at half-height, and the selectivity $(\alpha)$ was calculated according to $\alpha=\mu_{\mathrm{e} 1} / \mu_{\mathrm{e} 2}$ where $\mu_{\mathrm{e}}=\mu_{\mathrm{a}}-\mu_{\mathrm{EOF}}\left(\mu_{\mathrm{e}}\right.$ is the effective mobility, $\mu_{\mathrm{a}}$ is the apparent mobility and $\mu_{\mathrm{EOF}}$ is the electroosmotic mobility).

\section{RESULTS AND DISCUSSION}

The enantioresolution of the DNB derivatives of Phe and Leu was studied with the eight different SOs. Under the selected operating conditions, the tertiary quinuclidine moiety within the chiral SO is protonated and interacts with the negatively charged selectands (SA) to form neutral ion pairs moving with the cathodic electroosmotic flow. Thus the free and the complexed SA species have significantly different mobilities, a fact that gives rise to high enantioselectivity.

For the amino acid derivatives using the natural alkaloids as SOs, rather poor enantioseparations were observed. Higher mobility differences and selectivity values were obtained with the alkaloid derivatives (tBuCQN, tBuCQD, DNPCQN and cHexCQN). The additional substituent (tert-butyl, dinitrophenyl or cyclohexyl) in the SO and in particular the carbamate function, which may serve as hydrogen donor-acceptor, obviously has a favourable effect on enantioselectivity. For the DNB-Leu and the DNB-Phe enantiomers, the migration times $(t)$, the enantioselectivity $(\alpha)$ and the resolution $(R s)$ are presented in Table 1.

The following order in $\alpha$ values was observed for DNB-Leu: cHexCQN $>$ tBuCQD $>$ tBuCQN $>$ DNPCQN $>$ QD $>$ QN $>\mathrm{CN}>\mathrm{CD}$. The highest $\alpha$ (1.905) was obtained for the DNB-Phe enantiomers using cHexCQN and the highest Rs (80.0) for the same enantiomers with tBuCQD. In the presence of carbamoylated QN derivatives the migration order was reversed compared to QN. With $\mathrm{QN}, \mathrm{CD}$ and tBuCQD, the $(S)$-DNB-Leu enantiomer was migrating first, but with $\mathrm{QD}, \mathrm{CN}, \mathrm{tBuCQN}, \mathrm{DNPCQN}$ and $\mathrm{cHexCQN}$ the $(R)$-DNB-Leu enantiomer was migrating first. Using all these selectors, the enantiomers of DNB-Leu and DNBPhe were completely separated. For example, Fig. 2

Table 1. Enantioresolution of DNB-Leu and DNB-Phe with natural and derived alkaloids as selectors ${ }^{\text {a }}$

\begin{tabular}{|c|c|c|c|c|c|c|c|c|}
\hline \multirow[b]{2}{*}{ Chiral selector } & \multicolumn{4}{|c|}{ DNB-Leu } & \multicolumn{4}{|c|}{ DNB-Phe } \\
\hline & $t_{1}(\min )$ & $t_{2}(\min )$ & $\alpha$ & $R s$ & $t_{1}(\min )$ & $t_{2}(\min )$ & $\alpha$ & $R s$ \\
\hline QN & 20.03 & 21.55 & 1.057 & 5.5 & 19.90 & 20.55 & 1.025 & 2.6 \\
\hline QD & 22.52 & 24.80 & 1.060 & 8.3 & 14.93 & 15.54 & 1.028 & 3.6 \\
\hline $\mathrm{CN}$ & 20.12 & 21.75 & 1.055 & 7.0 & 19.08 & 19.82 & 1.027 & 3.1 \\
\hline CD & 21.59 & 23.06 & 1.046 & 5.3 & 22.34 & 23.07 & 1.022 & 2.7 \\
\hline tBuCQN & 15.97 & 36.14 & 1.572 & 64.3 & 16.94 & 35.66 & 1.504 & 61.1 \\
\hline tBuCQD & 19.84 & 51.32 & 1.783 & 78.3 & 16.28 & 38.03 & 1.759 & 80.0 \\
\hline DNPCQN & 19.52 & 23.98 & 1.151 & 17.3 & 18.30 & 22.60 & 1.158 & 13.4 \\
\hline cHexCQN & 19.55 & 44.58 & 1.787 & 66.9 & 16.82 & 40.78 & 1.905 & 56.2 \\
\hline
\end{tabular}

${ }^{a}$ Conditions as described in Material and Methods. 


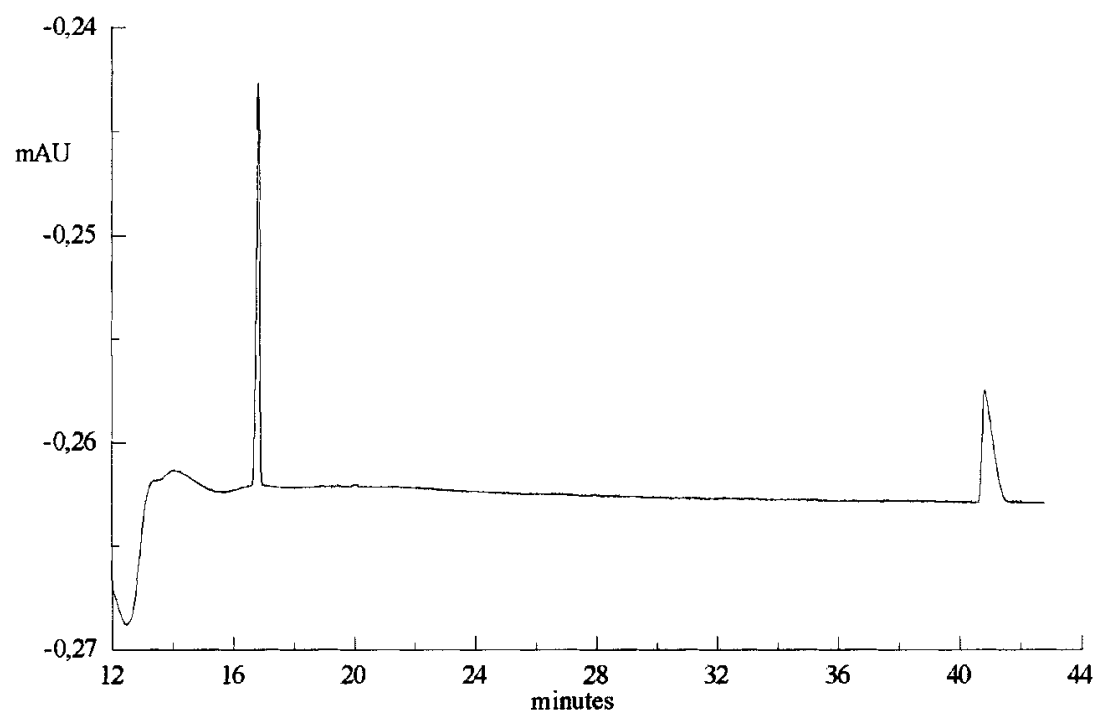

Figure 2. Enantioseparation of DNB-Phe with cHexCQN as chiral selector. Buffer: $100 \mathrm{mM}$ octanoic acid and $12.5 \mathrm{~mm}$ ammonia in methanol-ethanol (40:60) containing $10 \mathrm{mM}$ cHexCQN. Other conditions as described in Material and methods.

presents the electropherogram obtained for DNB-Phe with cHexCQN as SO.

\section{CONCLUSIONS}

A NACE system using a background electrolyte made from $100 \mathrm{mM}$ octanoic acid and $12.5 \mathrm{mM}$ ammonia in an ethanol-methanol mixture $(60: 40)$ was applied to the investigation of the potential of $\mathrm{QN}$, other cinchona alkaloids and derivatives for the enantioseparation of $\mathrm{N}$ protected amino acids. Particularly high enantiomeric resolution values (up to 80) were obtained for DNB amino acids using tert-butyl carbamoylated derivatives of quinine and quinidine as well as cyclohexyl carbamoylated quinine as chiral selectors.
In further work, the collected enantioselectivity values will be correlated with those obtained in HPLC using the same SOs imobilized onto silica as chiral stationary phase in order to apply this NACE method as a screening tool to evaluate the enantiodiscrimination potential of a larger set of newly developed chiral SOs derived from quinine and related alkaloids.

\section{REFERENCES}

Kleidernigg, O. P., Lämmerhofer, M. and Lindner, W., 1996. Enantiomer, 1:387-391.

Lindner, W., Lämmerhofer, M. and Maier, N. M., 1997. PCT/EP97/ 02888 ,

Piette, V., Lämmerhofer, M., Lindner, W. and Crommen, J., 1999. Chirality, 11:622-630. 